\title{
CAUSAS, MEDIOS Y ESTRATEGIAS DE AFRONTAMIENTO EN LA AGRESIÓN ONLINE EN ESCOLARES DE MURCIA (ESPAÑA)
}

\author{
Ana M. Gimenez Gualdo/Universidad de Murcia, España \\ Pilar Arnaiz Sanchez/Universidad de Murcia, España \\ Javier J. Maquilon Sanchez/Universidad de Murcia, España
}

\begin{abstract}
RESUMEN: Las situaciones de violencia entre iguales o bullying forman parte de la vida cotidiana de muchos escolares que viven sumidos en un círculo vicioso de humillaciones y vejaciones constantes por parte de sus compañeros dentro del centro educativo. Además, la fácil accesibilidad de los menores a las Tecnologías de la Información y la Comunicación (TIC), el escaso control y la conciencia imperante de impunidad en la red, dispone al ciberespacio como el lugar idóneo para violentar a otros iguales con la telefonía móvil e Internet. Este es el caso del cyberbullying que se está convirtiendo en una amenaza creciente para la integridad personal, psicológica y social de sus implicados. Aunque haya pasado una década desde que se comenzara a estudiar este tema, aún son escasas las investigaciones en España que indaguen en el conocimiento de sus causas, factores, roles implicados y consecuencias. Por ello, el presente artículo muestra los resultados encontrados al analizar las causas que ciberagresores y cibervíctimas atribuyen a la existencia de cyberbullying, las herramientas tecnológicas más utilizadas y las estrategias de afrontamiento de un grupo de escolares entre $6^{\circ}$ de Primaria a $1^{\circ}$ de Bachillerato de la Región de Murcia (España). Se concluye alertando de la necesidad de ahondar en el mayor conocimiento de esta problemática para prevenir sus peligrosas consecuencias en la vida presente y futura de nuestros estudiantes.
\end{abstract}

PALABRAS CLAVE: Agresividad. Bullying. Cyberbullying. Causas. Tecnologías de la Información y la Comunicación (TIC). Reacciones.

RESUMO: As situações de violência entre iguais ou bullying fazem parte da vida cotidiana de muitos escolares que vivem inseridos em um círculo vicioso de humilhações e vexações constantes por parte de seus colegas dentro do centro educativo. Ademais, a fácil acessibilidade dos menores às Tecnologias da Informação e a Comunicação (TIC), o escasso controle e a consciência imperante de impunidade na rede, colocam o ciberespaço como o lugar idôneo para violentar a outros iguais com a telefonia móvel e Internet. Este é o caso do cyberbullying que está se convertendo em uma ameaça crescente para a integridade pessoal, psicológica e social de seus implicados. Ainda que tenha passado uma década desde que se começasse a estudar este tema, ainda são escassas as investigações na Espanha que indaguem o conhecimento de suas causas, fatores, papéis implicados e consequências. Por isso, o presente artigo mostra os resultados encontrados ao analisar as causas que ciberagressores e cibervítimas atribuem à existência de cyberbullying, as ferramentas tecnológicas mais utilizadas e as estratégias de afrontamento de um grupo de escolares entre $6^{\circ}$ ano do Ensino Fundamental a $1^{\circ}$ ano do Ensino Médio da Região de Múrcia (Espanha). Conclui-se alertando sobre a necessidades de aprofundar no maior conhecimento desta problemática para prevenir suas perigosas consequências na vida presente e futura de nossos estudantes.

PALAVRAS-CHAVE: Agressividade. Bullying. Cyberbullying. Causas. Tecnologias da 
Informação e a Comunicação (TIC). Reações.

\section{INTRODUCCIÓN}

Desde que Dan Olweus iniciara la investigación en los años 70 en torno a la problemática de la violencia escolar entre iguales (bullying), a partir del suicido de varios jóvenes escandinavos como consecuencia del padecimiento de estas situaciones, son muchos los estudios dirigidos a indagar en el qué, cómo y por qué de estos comportamientos crueles entre pares. Sin embargo, la cotidianidad del bullying ha crecido en estas últimas décadas dejando de ser algo esporádico para convertirse en habitual (Cerezo, 2008). Por contra, el abordaje educativo que se ha ido prestando a los implicados no ha sido tan impetuoso y se ha reducido, en ocasiones, a actuaciones puntuales como la aplicación de una conducta más severa o el cambio de centro (COLLELL; ESCUDÉ, 2006).

Si la violencia en general vulnera los derechos humanos, la que se manifiesta entre los menores escolares es incluso más nefasta y destructiva para el presente y futuro de cada uno de ellos. El origen de estos comportamientos agresivos ha sido fuente de estudio desde finales del siglo XIX en la disciplina de la Psicología tratando de conocer qué factores predisponen a que una persona provoque daño ajeno. La naturaleza de estos determinantes puede ser tanto intrínseca (agresividad innata) como extrínseca (agresividad ambiental). En este último caso, los comportamientos agresivos se derivarían de la extrapolación de una violencia aprendida en el contexto familiar o social en el que se mueve el sujeto, o ser consecuencia de la manifestación de valores asumidos por los menores de la sociedad actual (ORTEGA; MORA; JÄGER, 2007). En cualquier caso, la escuela se sitúa como un escenario altamente peligroso para muchos menores que sufren en silencio situaciones de acoso permanentes.

Este hecho ha supuesto que tanto la escuela como la comunidad educativa en general se sitúen en el punto de mira en aras de ofrecer soluciones que minimicen las escabrosas consecuencias derivadas de la acción, visionado o sufrimiento del bullying. Por otro lado, la evolución constante de la sociedad y los cambios en los medios orientados al intercambio de información amparados por la inclusión masiva de las TIC en la vida de niños, jóvenes y adultos, exige de nuevas competencias, estrategias de control y habilidades sociales para su manejo y la relación interpersonal a través de estas. La gran afinidad existente entre los menores y las TIC sobre todo de aquellos conocidos como nativos digitales (PRENSKY, 2001), ha dado lugar a una nueva forma de acoso que supone un riesgo pasivo en el uso disfuncional de los medios tecnológicos (DEL RÍO; SÁDABA; BRINGUÉ, 2010).

Considerando la escasez de estudios previos en la Región de Murcia en torno a la incidencia del cyberbullying entre los escolares de Primaria y Secundaria, nos dispusimos a indagar en el mismo, atendiendo a diversas variables de las que destacamos en esta aportación: los motivos por los que agresores y víctimas justifican su implicación en el ciberacoso, las tecnologías utilizadas y las estrategias de afrontamiento de las víctimas ante el padecimiento de cyberbullying. 


\section{BULLYING: AGRESIVIDAD ENTRE IGUALES EN LAS AULAS}

Indagar en el origen de la conducta agresiva humana es complejo por la diversidad de factores que inducen a que una persona se comporte de forma vejatoria ante otra; pero una de las explicaciones cuando ésta se manifiesta en las dinámicas bullying se sitúa en la propia agresividad humana. Gerard (2002) la concibe como un estado emocional donde confluye el odio, la furia y la ira propiciando deseos de causar daño físico, sexual o psicológico a una persona, animal u objeto. Sin embargo, la atribución causal de la agresividad no puede ser simplista y concebirse como algo innato que no puede mejorarse sino que coincidimos con Alonso y Navazo (2002) en que se deriva de la interrelación de factores tanto de naturaleza biológica como ambiental. A nivel personal, la agresividad contra otros escolares puede deberse por ejemplo a: desajustes psicopatológicos (KUMPULAINEN; RÄSÄNEN; HENTTONEN, 1999), ausencia de conciencia (GARRIDO, 2007), presencia de trastornos de la infancia y la adolescencia tipo trastornos de atención e hiperactividad, disociales o negativistas desafiantes señalados en el DSM-IV-TR (APA, 2000) y CIE-10 (OMS, 2008), influencia del sexo (BORNTRAGER et al., 2009), procedencia cultural (TORREGROSA; INGLÉS; GARCÍA; RUIZ; LÓPEZ; ZHOU, 2010); o como señalan Cerezo (2008) y Pelegrín y Garcés (2008) ir ligado a un escaso autocontrol, generalización de la situación, baja tolerancia a la frustración, déficit en habilidades sociales, entre otras.

El ambiente familiar también puede condicionar y mucho el aprendizaje de actitudes agresivas y violentas hacia otros a partir del uso de la violencia entre sus miembros o la escasez de afecto y apego emocional. Además de la familia, otros factores ambientales son los señalados por Morales (2007) que incluye la temperatura, el ruido o el hacinamiento como precursores de comportamientos violentos. No podemos obviar, la influencia del grupo de iguales y la microcultura que se genera entre ellos (ORTEGA, 2005) que provoca que el sujeto carente de referencias o de la suficiente autonomía para saber decidir qué está bien y qué no, se deje llevar por el comportamiento antisocial de sus amigos. Por último mencionar el clima escolar. Está claro que un buen clima escolar va unido de forma directa con la reducción de la agresividad y violencia escolar, sin embargo no siempre el ambiente de aula y centro es el más propicio para la consecución de relaciones de convivencia que instan a la convivencia pacífica y el respeto mutuo. Si a ello le sumamos la falta de motivación y la aversión hacia la institución escolar la situación se torna más compleja. Por ende, el aula no debe ser sólo el lugar en el que aprender y enseñar sino que ha llegado el momento de comenzar a "educar los componentes y procesos psicológicos necesarios para desarrollar unas relaciones sociales e interpersonales competentes y respetuosas con los demás (...)” (TRIANES; GARCÍA, 2002, p. 176).

Entre las múltiples definiciones de bullying señalamos la de Olweus (2004) como precursor de esta línea de investigación que lo entiende cuando un alumno se convierte en víctima al estar expuesto de forma repetida en el tiempo a acciones negativas que provienen de otro alumno o alumnos. Cerezo (2009, 384) por su parte, lo caracteriza como "una forma de maltrato, normalmente intencionado, perjudicial y persistente de un estudiante o grupo de estudiantes, hacia otro compañero, generalmente más débil, al que convierte en su víctima habitual, sin que medie provocación y, lo que quizá le imprime el carácter más dramático, la incapacidad de la víctima para salir de esa situación, acrecentando la sensación de indefensión y aislamiento”.

Esta tipología de agresión no se manifiesta siempre de forma aparente a través de una violencia física, verbal o material, sino que en ocasiones se vuelve más sutil en forma de violencia psicológica (expansión de rumores, malos gestos, vejación de la autoestima, chantajes) o social/relacional (exclusión, ridiculizaciones, ignorancia) (ORTEGA; MONKS, 2006). Entre sus 
principales características, que ayudan a su detección, encontramos (DÍAZ-AGUADO; 2005; GÓMEZ et al., 2007; MENÉNDEZ, 2006; ORTEGA, 2006; MONTAÑÉS et al., 2009; OLWEUS, 2004):

- Acción agresiva persistente y continuada en el tiempo, que pasa de ser incidental a convertirse en sistemática;

- Hostilidad del agresor hacia la víctima;

- Generalmente ejercida entre un agresor respaldado por su grupo o de forma individual;

- Más presente la violencia física y directa entre chicos e indirecta (psicológica, exclusión) por parte de las chicas;

- La víctima se encuentra indefensa, aislada, sola y débil frente al agresor o agresores;

- Existe una importante relación desigual de poder entre agresor o víctima, lo que lleva a perpetuar el esquema fuerte-débil y, por ende, la ley de dominio y sumisión;

- Sensación permanente de miedo, frustración e incluso culpabilidad por parte de la víctima y desconfianza hacia los demás al no verse protegida o defendida por outros;

- Existencia de Ley del silencio tanto por parte de la víctima como del testigo por el miedo a ser descubiertos por el agresor, ignorados por los adultos (profesores, familia...) o emprender repulsas hacia el agresor;

- El agresor tiende a convertirse en el líder de su grupo o incluso de su clase;

- Los testigos tienden a colaborar en perpetuar la Ley del silencio negando tales situaciones o minimizando la actuación del agresor frente a la víctima;

- Multicausalidad, lo que implica multitud de factores de riesgo y/o predictores de la conducta violenta;

- Triángulo retroalimentado de violencia entre agresor-víctima-testigo;

- Común al comienzo de la adolescencia;

- Consecuencias devastadoras para sus implicados

El peligroso incremento que este fenómeno ha tenido en los últimos años y de forma concreta en España, sitúa su incidencia en porcentajes que deben ser tomados como preocupantes aunque no podemos fijar una cifra aproximada por la variedad de instrumentos que se han utilizado en cada uno de estos estudios. A continuación, resaltamos algunas de las investigaciones en España, y de la Región de Murcia en que se enmarca la investigación que se presenta:

1. Cruz Roja Juventud (2006): Con casi 700 alumnos de $1^{\circ}$ a $4^{\circ}$ de la ESO, los resultados desprenden que el 80\% afirmaba la existencia de bullying en sus centros educativos (Región de Murcia);

2. Oñate y Piñuel (2007): $44 \%$ de violencia escolar en $3^{\circ}$ de Primaria, $37 \%$ en $4^{\circ}$ de Primaria y un descenso conforme aumenta la edad escolar. Andalucía se situaba a la cabeza (28\%), seguida del País Vasco y Navarra (26\%), Asturias y Cantabria (24\%), mientras que Murcia se encontraba por debajo del nivel medio;

3. Defensor del Pueblo (2007): 27\% de agresión verbal, 26\% de agresión indirecta y 11\% de exclusión social; 
4. Cerezo (2009): Los chicos siguen siendo los más implicados en dinámicas bullying como agresores frente a las chicas que mantienen más su posición como víctimas. La incidencia del bullying por niveles nos hace ver el crecimiento significativo en la última década con un 32\% en Educación Primaria y un 34\% en Secundaria. De nuevo la agresión verbal sigue siendo la más normalizada, seguida de la violencia física más común en Primaria y la exclusión social en Secundaria;

5. Garaigordobil y Oñederra (2010): La agresión más común entre los escolares de primaria y secundaria del País Vasco es la de tipo verbal, lo que coincide con otros estudios previos, siendo más común entre los niños el rol de agresores y el lugar de acoso, el patio. El porcentaje de bullying en el último ciclo de educación primaria fue mayor (6\%) que en secundaria (4\%).

Considerando la perversidad que suponen estas dinámicas, es imprescindible incrementar la implicación y participación activa de la familia, el profesorado y el propio alumnado en aras de prevenir y actuar contra el aumento del acoso entre escolares (MAQUILÓN; GIMÉNEZ; HERNÁNDEZ; GARCÍA, 2011a; 2011b).

\section{CYBERBULLYING: LA AGRESIÓN ONLINE DEL SIGLO XXI}

Cuando el acoso escolar deja de circunscribirse al ámbito escolar con comportamientos relativamente aparentes y queda mediatizado por las tecnologías que serán el medio para continuar acosando a la víctima, se da paso a una nueva forma de violencia entre pares conocida como cyberbullying (BELSEY, 2005). Estas dinámicas incluyen comportamientos crueles entre iguales a través del envío de mensajes, vídeos, mails con contenidos dañinos o la puesta en práctica de diversas formas de crueldad social a través de Internet y las tecnologías digitales (WILLARD, 2006). En términos similares, Smith, Mahdavi, Carvalho y Tippett (2006) lo conciben como la agresión intencional ejercida de forma grupal o individual haciendo uso de las formas electrónicas de contacto, de forma repetida y constante, sobre una víctima que no puede defenderse fácilmente. Ferdon y Hertz (2009) lo entienden como cualquier tipo de acoso o bullying (burlas, expansión de rumores falsos, denigración, agresión verbal) generado a través del email, salas de chat, mensajería instantánea, páginas web, mensajes de texto, videos o imágenes subidas a una web o enviadas por teléfono. En general, ahora la violencia cara a cara se sustituye por el ordenador confiriéndole al acoso mayor sutileza e invisibilidad. En la Tabla 1 pueden consultarse las principales formas de presentación de esta forma de acoso atendiendo al medio y acción perpetrada.

Tabla 1: Tipologías de cyberbullying (elaboración própria).

Autor

Acciones

Medios

Aftab (2006) Acoso cibernético directo Email, mensajes de texto (SMS), robo de contraseñas, páginas web, envío de imágenes por email o teléfono móvil, votaciones por Internet, juegos online, envío de virus, envío de correos pornográficos y/o basura, envío de mensajes multimedia (MMS) pornográficos y/o basura, suplantación de la personalidad (usuario/perfil)

Acoso cibernético por Cualquiera de los anteriores medios para pirateo de la cuenta delegación/ de la víctima y envío de correos difamatorios a los amigos de 


\begin{tabular}{|c|c|c|}
\hline & indirecto & esta, bloqueo de cuentas de la víctima... \\
\hline \multirow{6}{*}{$\begin{array}{l}\text { Willard (2005; } \\
\text { 2006) }\end{array}$} & Insultos electrónicos & Cualquier medio tecnológico \\
\hline & Hostigamiento & $\begin{array}{l}\text { A través de cualquier medio tecnológico, supone el envío de } \\
\text { mensajes ofensivos reiterados a la víctima }\end{array}$ \\
\hline & Denigración & $\begin{array}{l}\text { A través de cualquier medio tecnológico, sucede por la } \\
\text { expansión de rumores inciertos para dañar la reputación de } \\
\text { otro/s }\end{array}$ \\
\hline & Desvelamiento & $\begin{array}{l}\text { A través de cualquier medio tecnológico, supone la revelación } \\
\text { y difusión de información personal o íntima de la víctima } \\
\text { utilizando la confianza depositada y su amistad }\end{array}$ \\
\hline & Exclusión u ostracismo & $\begin{array}{l}\text { Utilizando cualquier medio sucede a partir de la exclusión de la } \\
\text { víctima de un dominio de red, juego online, salas de chat, etc., } \\
\text { o eliminación de la víctima de una lista de contactos }\end{array}$ \\
\hline & Ciberpersecución & $\begin{array}{l}\text { Uso de herramientas tecnológicas para perseguir a otra persona } \\
\text { de forma continua hostigándole y amenazándole. Se diferencia } \\
\text { del hostigamiento en que utiliza más bien las amenazas }\end{array}$ \\
\hline $\begin{array}{l}\text { Kowalski, Limb } \\
\text { y Agatston }(201\end{array}$ & $\begin{array}{l}\text { Manteniendo la } \\
\text { clasificación de Willard, } \\
\text { añaden la paliza feliz } \\
\text { ("happy slapping”) }\end{array}$ & $\begin{array}{l}\text { A través del móvil el/los agresor/es graba/graban una situación } \\
\text { de agresión física o psicológica ejercida contra otro igual para } \\
\text { posteriormente subirlo a Internet y difundirlo por las redes } \\
\text { sociales, Youtube, etc. }\end{array}$ \\
\hline
\end{tabular}

El cyberbullying es un fenómeno relativamente reciente que comienza a ser investigado a principios del año 2000 en Estados Unidos con Finkelhor, Mitchell y Wolak (2000). Estos autores señalaban alrededor de un 6\% de cibervictimización a través de herramientas como el Messenger, salas de chat y el correo electrónico. Unos años más tarde, Beran y Li (2005) realizaron una investigación con jóvenes entre 12-15 años obteniendo una incidencia de 69\% de cibertestigos, 21\% de cibervictimización y 3\% de cyberbullying. Además encontraron esa conexión entre bullying y cyberbullying a partir de que más de la mitad de los encuestados afirmaron ser también víctimas del bullying tradicional. Por su parte, Li (2006) aborda la cuestión de las diferencias entre sexos, encontrando una mayor implicación de chicos como agresores online y de chicas como cibervíctimas. Lenhart, en 2007, confirma que uno de cada diez menores ha sido víctima del mismo encontrando similitudes de implicación por sexo como Li (2006).

Una visión general de algunas de las principales investigaciones nacionales e internacionales realizadas en los últimos años sobre esta línea de investigación pueden consultarse en Garaigordobil (2011) y Álvarez et al. (2011). Entre los destacados en España señalamos los estudios en 2010 y 2011 del Observatorio de la Seguridad de la Información. En el primero referido a la privacidad en los móviles por los menores españoles entre 6-10 años, el cyberbullying aparece como un riesgo a considerar con un 5.9\% de cibervictimización. En el estudio de 2011 sobre el uso de los smartphones entre los menores, el cyberbullying aparece como un importante riesgo (2.5\% de cibervictimización y $0.8 \%$ de agresión), además del consumo excesivo, dependencia, amenazas a la privacidad, acceso a contenidos inapropiados, grooming (solicitud de índole sexual de un adulto hacia un menor), riesgo económico, fraude, riesgo de carácter técnico y sexting, entendido este último como la producción e intercambio de imágenes de contenido sexual explícito de uno mismo o de otros a través de la telefonía móvil o el ordenador (McLAUGHLIN, 2010). En el estudio de Garmendia, Garitaonandia, Martínez y Casado (2011) se obtuvo un 5\% de cyberbullying, siendo los medios más utilizados las redes sociales y la mensajería instantánea. A nivel de la la Región de Murcia, ya se ha iniciado la investigación en torno a esta temática, de la que a continuación resaltamos algunos de los resultados obtenidos aunque puede ampliarse la información en: Giménez y Maquilón (2011), Maquilón, Giménez, Hernández y García (2011a, 2011b), Giménez (2012), 
Giménez, Arnaiz y Maquilón (2013). En esté último estudio además se establece la conexión del cyberbullying con el proceso de exclusión social y educativa que van a padecer tanto la víctima, como a veces el agresor, línea de investigación que actualmente estamos desarrollando.

En general, el cyberbullying se sucede no sólo porque exista intencionalidad de causar daño ajeno según las tipologías de agresores (WIREDSAFETY; AFTAB, 2008), sino que puede ir unido a una adicción previa a las nuevas tecnologías.

El consumo excesivo que los menores hacen de las TIC repercute en que pasen de ser elementos de trabajo y ocio a ser una necesidad vital. Es precisamente en el hogar donde los jóvenes establecen un mayor tiempo de relación con las TIC, una relación en la mayoría de los casos individual y personal (Ballesta y Cerezo, 2011). Si a ello le sumamos el escaso control por parte de los padres sobre el acceso y tiempo que los menores pasan frente al ordenador, la televisión o el móvil, la situación se agrava aún más permitiendo que muchos jóvenes desarrollen conductas adictivas hacia estos medios. Estudios como el de Echeburría, Labrador y Becoña (2009) o los del Observatorio de la Seguridad de la Información (2010; 2011) confirman esta situación.

Tanto las características del bullying como las propias del cyberbullying (Tabla 2) se sustentan bajo un círculo vicioso en continua retroalimentación lo que indica que ante la Ley del Silencio propia de agresores, víctimas y testigos, estos comportamientos agresivos dentro y fuera de la escuela se suceden uno tras otro, cada vez más graves y de consecuencias más devastadoras. (LENHART, 2007; WILLARD, 2005; 2006).

Tabla 2: Características del cyberbullying (elaboración própria).

Naturaleza indirecta que acrecienta la potencialidad mayor de la agresividad

Mayor persistencia en el tiempo

Mayor invisibilidad = menor empatía

Inseguridad constante de la víctima al trasladarse el acoso a cualquier tiempo y lugar

Anonimato del agresor

Tipos según los medios que se utilicen y las acciones que se realicen (WILLARD, 2005; 2006)

Impunidad del agresor por la invisibilidad que ofrecen las nuevas tecnologías

Mayor inadvertencia ante los adultos

Triángulo de la violencia

Consecuencias aún más devastadoras

Expansión ilimitada a una gran audiencia

Correlación entre bullying tradicional-cyberbullying

Es importante señalar que para que exista cyberbullying éste debe ser protagonizado por menores (GARAIGORDOBIL, 2011) ya que la presencia de algún adulto estaría determinando otra forma de acoso cibernético conocido como grooming. Kowalski, Limber y Agatston (2010) establecen una comparativa entre los fenómenos bullying y cyberbullying. Ambas formas de acoso comparten: (1) la agresividad, (2) la permanencia en el tiempo y (3) la relación de desequilibrio entre el agresor y la víctima. Sin embargo, se diferencian en cuatro elementos que confieren al 
cyberbullying un mayor peligro: (1) el desconocimiento/anonimato del agresor, (2), la accesibilidad que provoca que la víctima se encuentra atormentada las 24h del día los 7 días de la semana, (3) miedo al castigo, por lo que las víctimas ocultan su padecimiento para que no les restrinjan el móvil o Internet, (4) espectadores ocasionales que de forma voluntaria o involuntaria conocen de estas situaciones.

La naturaleza electrónica del cyberbullying provoca que estemos hablando de un acoso indirecto que se presenta de múltiples formas y a través de diversos medios (Tabla 2). Cualquier de estas formas de cyberbullying lleva ligado una serie de riesgos a los que se ven expuestos sus implicados y sobre todo las víctimas. Willard (2006) distingue entre: (1) riesgos relacionados con la sexualidad; (2) comunidades online inseguras; (3) grupos online peligrosos; y (4) otros riesgos. En cualquier caso, poner fin al cyberbullying es complicado aún mas cuando se desconocen los motivos, causas y demás elementos que condicionan a los menores a utilizar la red como medio para vejar a otros. Para concluir, coincidimos con Mora (2008, p. 69) en que "el cyberbullying es un problema que debe ser tratado y los centros y las familias no deben mirar hacia otro lado, de lo contrario los efectos sobre la vida de los centros y sobre los alumnos que desgraciadamente se ven involucrados en ellos se pueden ver aumentados”.

\section{MÉTODOLOGÍA}

\subsection{Objetivos}

Ante la escasez de estudios previos en la Región de Murcia que indaguen en el fenómeno cyberbullying entre los escolares, nos propusimos realizar un primer acercamiento a estas dinámicas estableciendo como propósito general el analizar la incidencia del cyberbullying entre los estudiantes del último ciclo de Educación Primaria y ESO de nuestra provincia desde la perspectiva del agresor y de la víctima. Entre los objetivos específicos que nos marcamos, en el presente trabajo damos respuesta a los tres siguientes:

1. Analizar los motivos por los que agresores y víctimas justifican su implicación en cyberbullying según el sexo;

2. Describir las tecnologías utilizadas en cyberbullying según el sexo de agresores y víctimas;

3. Valorar las reacciones de las víctimas ante el cyberbullying.

\subsection{Participantes}

Los participantes invitados fueron un total de 816 estudiantes del último ciclo de Educación Primaria ( $5^{\circ}-6^{\circ}$ curso), ESO $\left(1^{\circ}\right.$ a $\left.4^{\circ}\right)$ y $1^{\circ}$ de Bachillerato de ocho centros educativos de titularidad pública y privada concertada de la Región de Murcia. La selección muestra fue de carácter aleatorio por conglomerados con una muestra final de 783 escolares durante los meses de Febrero a Mayo de 2011. 


\subsection{Instrumento}

Para la recogida de la información se aplicó el cuestionario Cyberbull (GIMÉNEZ; ARNAIZ; MAQUILÓN, 2013), diseñado de forma específica para esta investigación. Tras la revisión del mismo mediante un primer pilotaje, se rediseñó y adaptó reduciendo el número de preguntas para lograr un total de 6 preguntas que ahondan en las variables: causas y medios utilizados por agresor y víctima en cyberbullying (4), reacción de la víctima ante cyberbullying (1) y percepción diferenciada entre bullying y cyberbullying. Otras variables presentes fueron el nivel educativo, sexo, edad, nacionalidad, titularidad del centro, supervisión paterno/materna y resultados académicos. La fiabilidad (alfa de Cronbach) de este instrumento es aceptable (.59).

\subsection{Resultados}

En el primer objetivo analizamos las causas por las que tanto agresores como víctimas justifican su participación en cyberbullying. Del total de encuestados, se obtuvo un $9,8 \%$ de agresores con un ligero mayor porcentaje de niños (55.3\%) que de niñas (44.7\%). La Fig. 1 indaga en los motivos que ambos roles justifican de su participación en el ciberacoso. Observamos que el principal motivo tanto en agresores niños como niñas para acosar online es la "provocación”, esto es, entienden que la víctima les provoca o en realidad así es, con apenas un $1 \%$ más de niñas (58.8\%) que niños (57.2\%). El segundo motivo más representado en el caso de los niños es tanto la “diversión” como la percepción de “debilidad” de la víctima. Éste último motivo también es el segundo más elegido por las agresoras.

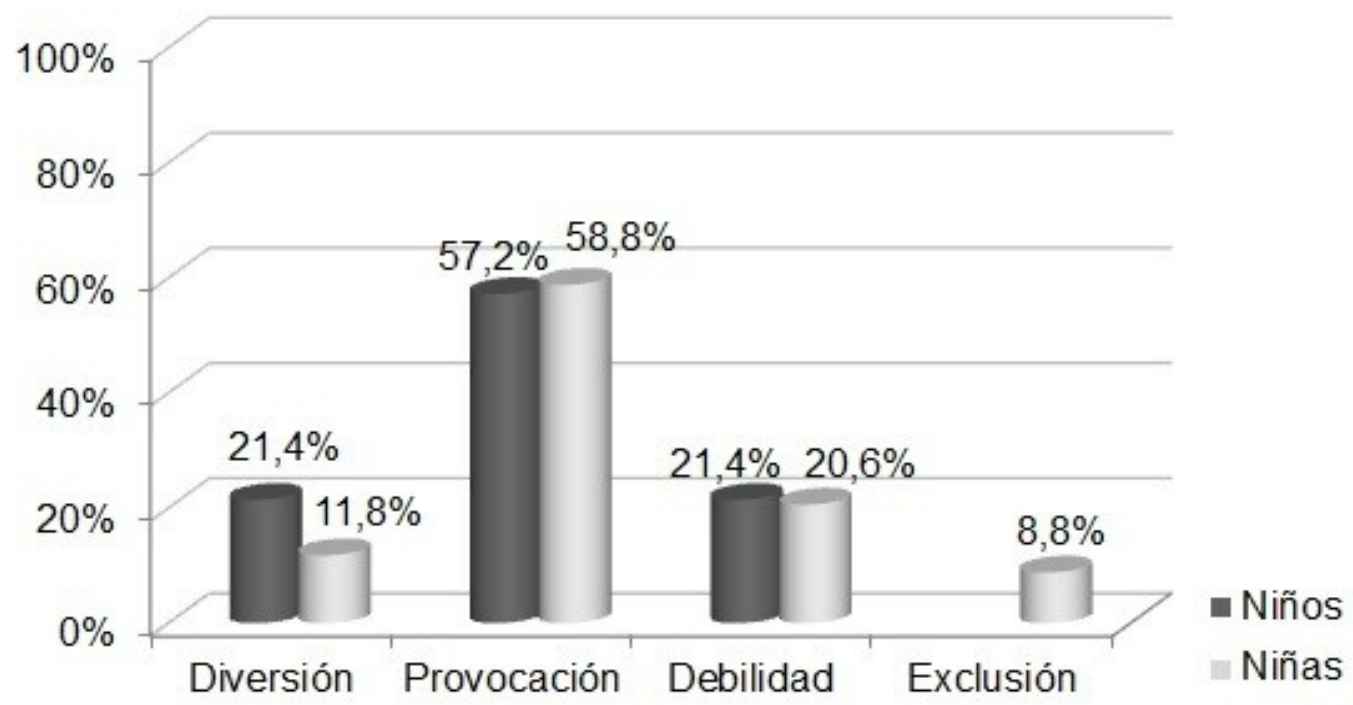

Gráfico 1: Motivos de implicación en cyberbullying según ciberagresores por sexo.

Sin embargo, ellas justifican, aunque en cuarto lugar (8.8\%) que ciberagreden a las víctimas porque son diferentes, generando con ello su exclusión.

En el caso de las víctimas la incidencia asciende al 11.9\% con un ligero mayor número de niñas (50.5\%) que de niños (49.5\%). En la Fig. 2 encontramos un quinto motivo hasta ahora no presente en los agresores como es la "revancha", es decir, reciben cyberbullying y lo ejercen, 
convirtiéndose así en víctimas activas (15.2\% niños; $8.5 \%$ niñas). El principal motivo ahora por el que las víctimas perciben que les violentan es porque al agresor/a les supone “diversión” con porcentajes muy similares entre sexos. La segunda causa va a ser en ambos casos la autopercepción de “debilidad”, mayor en niñas (21.3\%) que en niños (19.6\%).

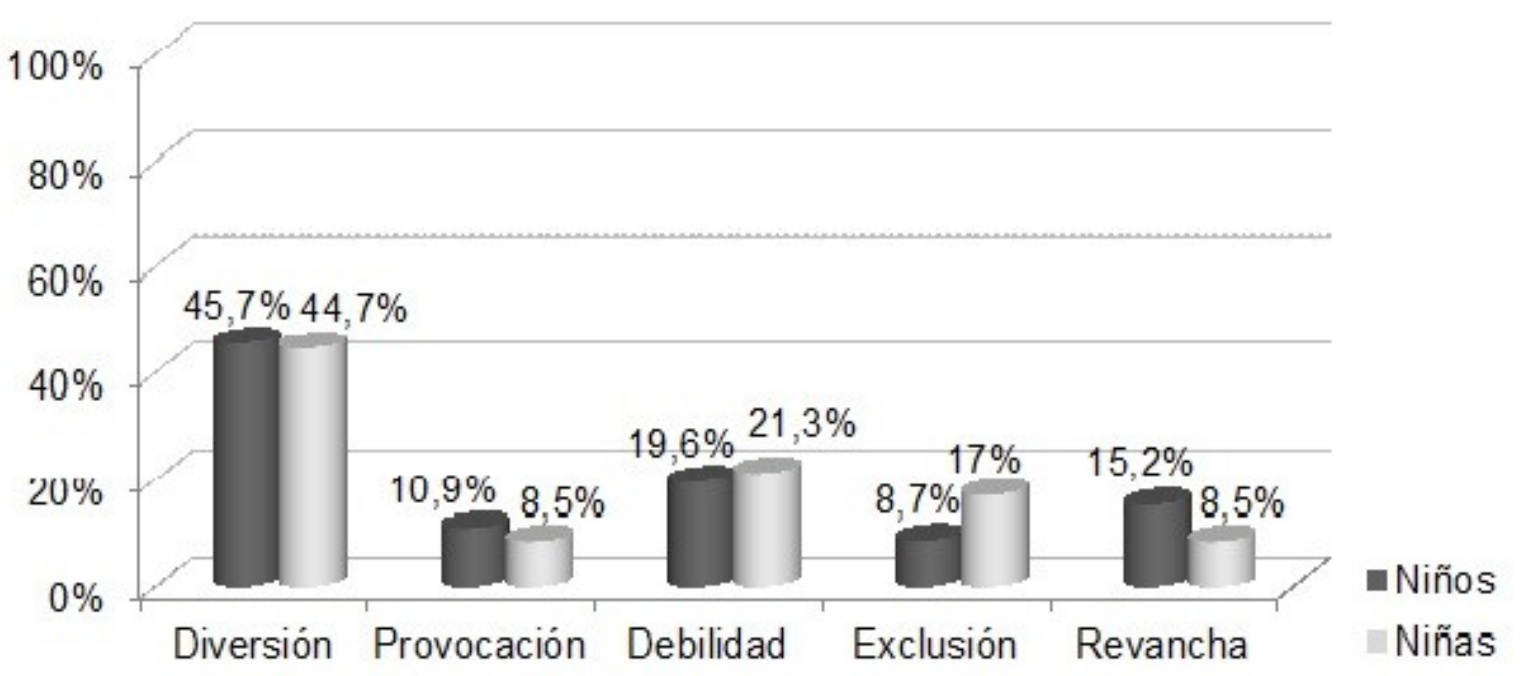

Gráfico 2: Motivos de implicación en cyberbullying según cibervíctimas por sexo.

Respecto al segundo objetivo referido a los medios y/o aplicaciones utilizadas en cyberbullying, se observa una similitud generalizada en cuanto a las herramientas que se utilizan y al número de las mismas tanto en chicas como en chicos. Desde la perspectiva de los agresores observamos (Fig. 3) que los chicos se decantan por un uso múltiple de medios, hasta siete, para ciberacosar a la víctima. Estos medios son: los mensajes de texto (SMS), mensajes multimedia (MMS), mensajería instantánea (Messenger), vídeos, Youtube, emails y las redes sociales (RS), con un 54.2\% frente al 40\% de chicas. Por su parte, las niñas prefieren el Messenger (44\%) como la principal herramienta para agredir digitalmente a sus víctimas. Con porcentajes menores encontramos la multiplicidad de cuatro tecnologías y en último término los SMS (4\% en chicas; $5.1 \%$ en chicos).

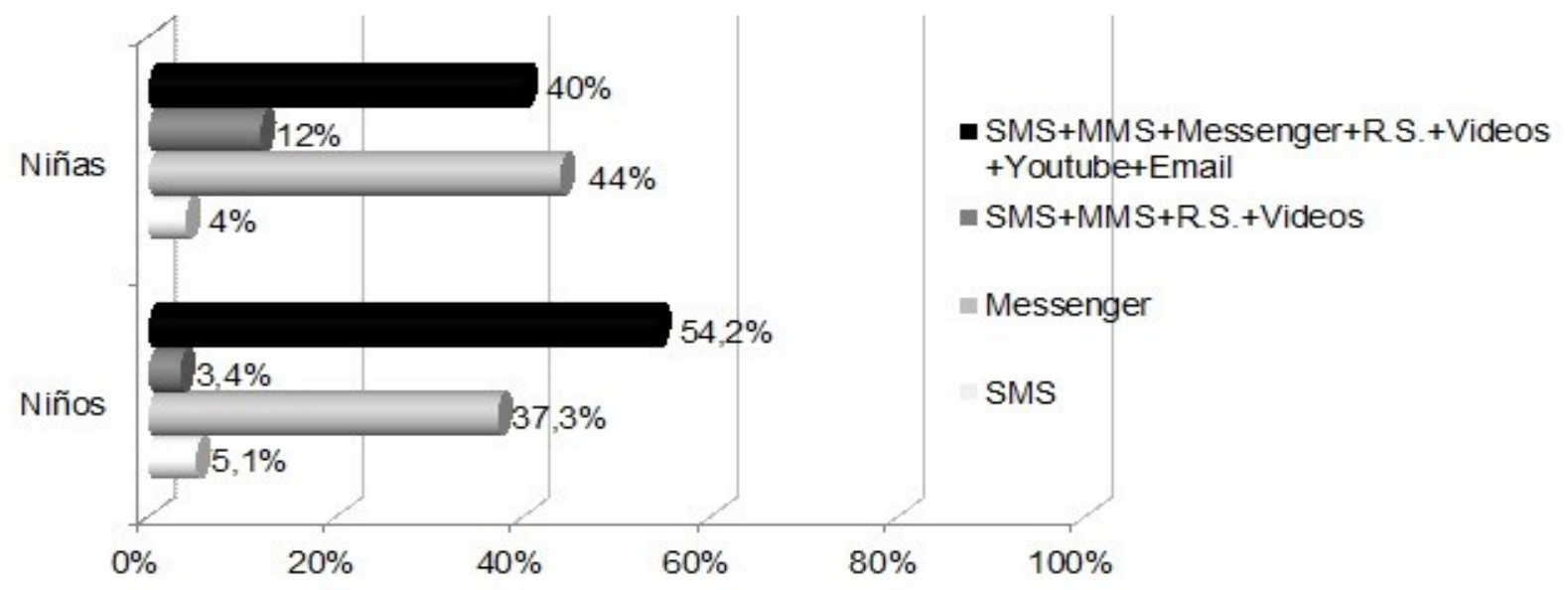

Gráfico 3: Diferencias por sexo en las tecnologías utilizadas por ciberagresores. 
Cuando las víctimas reciben el ciberacoso (Fig. 4), tanto niñas (33.3\%) como niños (31.5\%) lo van a hacer a través de la multiplicidad de hasta siete tecnologías diferentes, aunque en el caso del sexo masculino existen un mayor porcentaje de víctimas que lo van a ser a través de las redes sociales (34.1\%). La mensajería instantánea o Messenger también está altamente representado con un $23.5 \%$ en chicas víctimas y un $22.9 \%$ en chicos. De nuevo, los SMS son los menos utilizados en ambos casos.

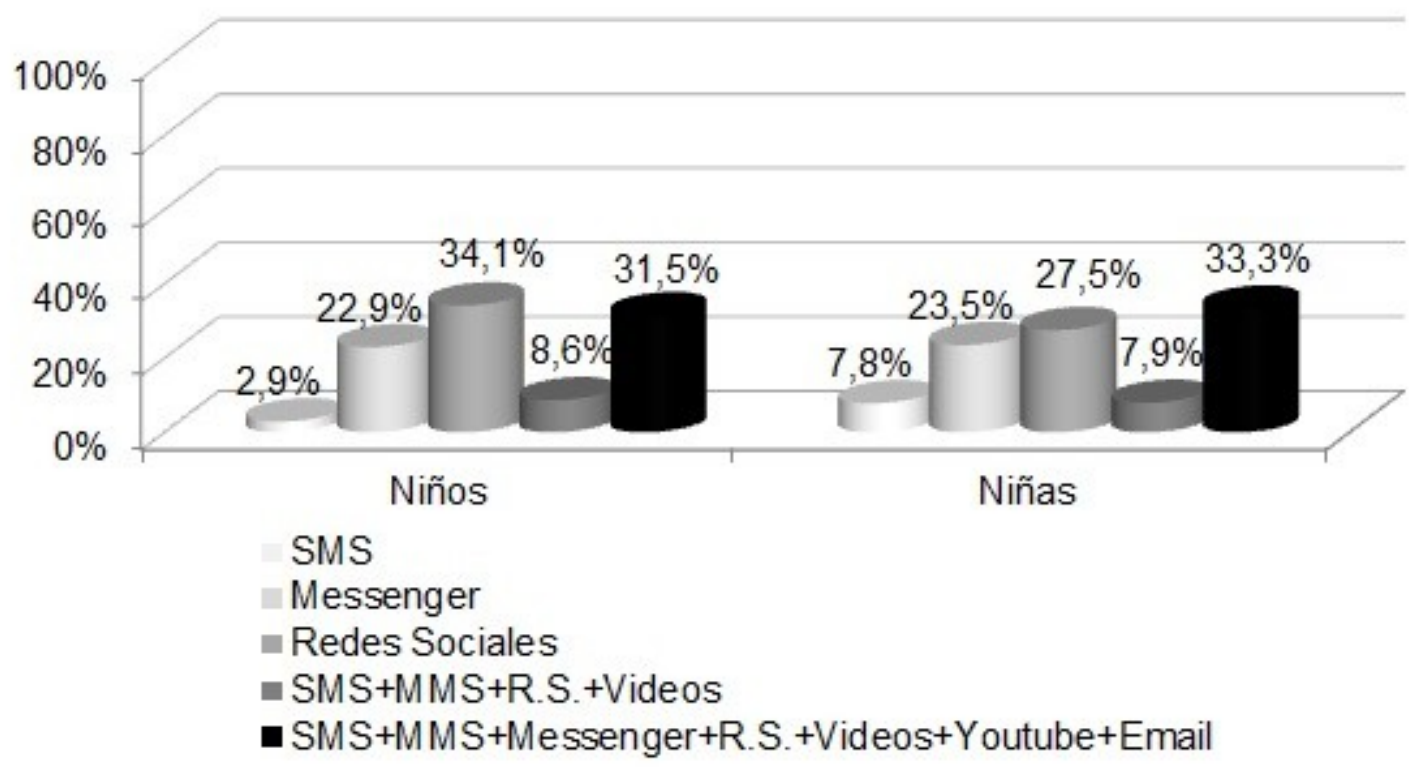

Gráfico 4: Diferencias por sexo en las tecnologías utilizadas según las cibervíctimas.

En último lugar, el objetivo tercero nos ofrece la posibilidad de conocer las principales estrategias de afrontamiento utilizadas por las cibervíctimas ante el padecimiento de agresión online. Entre las respuestas dadas, en ambos sexos (56.1\% chicos, $40.8 \%$ chicas) se opta por contestar y contraatacar al agresor en primer término convirtiéndose con ello en lo que se conoce como víctimas agresivas y/o provocativas. En segundo lugar, los niños eligen no hacer nada (17.5\%) seguido de comentarlo con las amistades (15.8\%), mientras que en el caso del género femenino existe una mayor tendencia a decírselo a la familia (23.9\%) y en tercer lugar, compartir estas vivencias con las amistades (16.9\%). Por su parte, acudir al profesorado para buscar apoyo o ayuda sigue siendo la última estrategia que tanto niños $(5.3 \%)$ como niñas $(4.2 \%)$ víctimas de cyberbullying realizan para solventar tales situaciones.

\section{DISCUSIÓN Y CONCLUSIONES}

El peligroso aumento de las dinámicas cyberbullying entre los escolares de último ciclo de Educación Primaria, ESO y Bachillerato, proyecta la necesidad de más investigaciones que ahonden en el conocimiento de las variables implicadas en tales comportamientos de vejación tecnológica. Sin intención de generalizar, los resultados obtenidos pretenden dar la voz de alarma a padres, alumnos y profesores de la Región de Murcia, en aras de comenzar a trabajar activamente en su detección, prevención y tratamiento. 
Los datos resultantes muestran una participación ligeramente mayor de los escolares murcianos niños como agresores, mientras que las niñas participan en mayor medida como víctimas, lo que confirma los resultados de estudios previos (LI, 2006; LENHART, 2007).

En relación a las causas por las que los agresores justifican ciberacosar a sus víctimas, la provocación se sitúa en primer lugar en ambos sexos. Esto debe tomarse en consideración indagando más de lleno en el porqué de este motivo, ya que el comportamiento de los niños y niñas bully no sucede tanto porque otros les provoquen sino porque así lo interpretan ellos, existiendo una distorsión cognitivo-perceptiva (DELGADO; CONTRERAS, 2008), o por su personalidad fácilmente provocable (GÓMEZ et al, 2007). Por su parte las víctimas justifican sentirse ciberagredidas porque perciben que el agresor/a se divierte con ello o por su parte, porque se autoperciben como débiles e inferiores. El hecho de que la diversión esté implícita en estas dinámicas debe hacernos reflexionar sobre la libertad de acceso de los menores a las tecnologías, la psicología implícita de felicidad y satisfacción con el mal ajeno o la sensación de aburrimiento descrita por Varjas, Talley, Meyers, Parris y Cutts (2010). La percepción personal de las víctimas como débiles frente al agresor, responde a sus propias características personales ligado ella a su escasa ascendencia social (CEREZO, 2002) o a sentimientos de inferioridad e inseguridad (GARAIGORDOBIL; OÑEDERRA, 2010).

Sobre las tecnologías utilizadas en el cyberbullying, tanto en los agresores como en las víctimas los mayores porcentajes están representados por el uso múltiple de herramientas. Eso puedo hacernos pensar en una de las características propias del cyberbullying en comparativa al bullying que es precisamente la temporalidad de la intimidación, es decir, que puede ocurrir a través de diferentes medios, las 24 horas del día, 7 días a la semana, incluso de forma simultánea. La segunda herramienta más utilizada para acosar online a la víctima es el Messenger, preferida más por las chicas. En la victimización volvemos a encontrar la misma situación, esto es, el acoso se recibe a través de diversos canales y entre ellos, las redes sociales se sitúan en segundo lugar como la herramienta por la que las víctimas niños o niñas son ciberagredidas.

Por último, el análisis de las estrategias de afrontamiento ante el cyberbullying sugiere que contraatacar y convertirse así en víctima agresiva o proactiva (ESTÉVEZ; JIMÉNEZ; MORENO, 2010) es en primer orden la respuesta ofrecida por las víctimas. En segundo término se sitúa la familia según el testimonio de las niñas, mientras que los niños optan por una actuación pasiva de no hacer nada. Ambos sexos coinciden en recurrir en tercer lugar a las amistades para compartir con ellas las experiencias de acoso online, quedándose la figura del profesorado como último recurso al que acudir en busca de ayuda o apoyo. Esto debe hacernos reflexionar sobre la actitud, en ocasiones, pasiva y desconocedoras de estas situaciones del profesorado y la familia ante un fenómeno tan perjudicial y maligno como es en cyberbullying en edades tan tempranas como los 11-12 años. Surge así la urgente necesidad de empezar a formar a familias y profesorado en el conocimiento de estas nuevas formas de acoso escolar para su detección y tratamiento educativo durante los tiempos escolares y en el hogar, generando cauces de confianza para con los alumnos que eviten la peligrosa propagación de estas agresiones encubiertas. En conclusión, optamos por la necesidad de seguir generando esfuerzos tanto para ampliar las investigaciones en nuestra región que nos ayuden a conocer de forma más profunda de este fenómeno, como, sobre todo, en empezar a sensibilizar al alumnado y el profesorado en aras de prevenir su expansión entre los escolares. 


\section{REFERENCIAS}

AFTAB, P. Stop Cyberbullying: How cyberbullying works? 2006. Disponible en: <http://www.stopcyberbullying.org/how it works/index.html>. Acceso el: 28 ago. 2013.

ALONSO, J. y NAVAZO, M. A. La agresividad escolar y su relación con el rendimiento escolar. Revista Electrónica Interuniversitaria de Formación del Profesorado, 5 (1), p. 1-5, 2002.

ÁlvArEZ, D., NÚÑEZ, J. C., ÁlVAREZ, J. L., DOBARRO, A., RODRÍGUEZ, C. y GONZÁLEZ, P. Violencia a través de las tecnologías de la información y la comunicación en estudiantes de secundaria. Anales de psicología, 27 (1), p. 221-231, 2011.

AMERICAN PSYCHIATRIC ASSOCIATION (APA). Diagnostic and Statistical Manual of Mental Disorders Fourth Edition, Text Revision (DSM-IV-TR). Arlington: American Psychiatric Association, 2000.

BALLESTA, J. y CEREZO, M. C. Familia y escuela ante la incorporación de las tecnologías de la información y la comunicación. Revista Educación XX1. 14 (2), p. 133-156, 2011.

BELSEY, B. Cyberbullying: An emerging threat to the "always on" generation, 2005. Disponible en: <http://www.cyberbullying.ca/pdf/Cyberbullying Article by Bill Belsey.pdf $>$. Acceso el: 28 ago. 2013.

BERAN, T, y LI, Q. Cyber-harrasment: a study of a new method for an old behaviour. Journal of Educational Computing Research. 32 (3), p. 265-277, 2005.

BORNTRAGER, C., DAVIS, J. L., BERNSTEIN, A., y GORMAN, H. A cross-national perspective on bullying. Child \& Youth Care Forum. 38, p. 121-134, 2009.

CEREZO, F. El bullying y su relación con las actitudes de socialización en una muestra de adolescentes. Revista Electrónica Interuniversitaria de Formación del Profesorado. 5 (1), 2002.

CEREZO, F. Acoso escolar. Efectos del bullying. Boletín de la Sociedad de Pediatría de Asturias, Cantabria y Castilla y León. 48 (206), p. 353-358, 2008.

CEREZO, F. Bullying: análisis de la situación en las aulas españolas. International Journal of Psychology and Psychological Therapy. 9 (3), p. 383-394, 2009.

COLLELL, J.; ESCUDÉ, C. El acoso escolar: un enfoque psicopatológico. Anuario de Psicología Clínica y de la Salud. 2, p. 9-14, 2006.

CRUZ ROJA JUVENTUD. Estudio sobre las situaciones de violencia entre los adolescentes del municipio de Murcia. Murcia: Ayuntamiento de Murcia, Servicio de Juventud, 2006.

DEFENSOR DEL PUEBLO. Violencia Escolar: El maltrato entre iguales en la Educación Secundaria Obligatoria 1999-2006. Madrid: Defensor del Pueblo, 2007. 
DELGADO, B. y CONTRERAS, A. Desarrollo social y emocional. En: DELGADO, B. (Coord.). Psicología del desarrollo: desde la infancia a la vejez. Madrid: McGraw Hill, 2008. p. 35-66.

DEL RÍO, J., SÁDABA, C. y BRINGUÉ, X. Menores y redes ¿sociales?: de la amistad al cyberbullying. Revista de Estudios de Juventud, 10 (88), p. 115-129, 2010.

DÍAZ-AGUADO, M. J. La violencia entre iguales en la adolescencia y su prevención desde la escuela. Revista Psicothema. 17 (4), p. 549-558, 2005.

ECHEBURRÍA, .E, LABRADOR, F. J.; BECOÑA, E. Adicción a las nuevas tecnologías en adolescentes y jóvenes. Madrid: Pirámide, 2009.

ESTÉVEZ, E.; JIMÉNEZ, T.; MORENO, D. Cuando las víctimas de violencia escolar se convierten en agresores: «¿Quién va a defenderme?». European Journal of Education and Psychology. Vol. 3, Núm. 2, p. 177-186, 2010.

FERDON, D.; HERTZ, M. F. Electronic media and youth violence: A CDC issue brief for researchers. Atlanta: Centers for Disease Control, 2009.

FINKELHOR, D.; MITCHELL, K. J.; WOLAK, J. Online victimization: A report on the nation's youth. Alexandria, VA: National Center for Missing and Exploited Children, 2000.

GARAIGORDOBIL, M. Y OÑEDERRA, J. A. La violencia entre iguales. Revisión teórica y estrategias de intervención. Madrid: Pirámide, 2010.

GARAIGORDOBIL, M. Prevalencia y consecuencias del cyberbullying: Una revisión. International Journal of Psychology and Psychological Therapy. 11 (2), p. 233-245, 2011.

GARMENDIA, M.; GARITAONANDIA, C.; MARTÍNEZ, M.; CASADO, M. A. Riesgos y seguridad en Internet: Los menores españoles en el contexto europeo, 2011. Disponible en: <http://www.sociologia.ehu.es/s0018eukidsct/es/contenidos/noticia/eukids informe 280311/es not/adjuntos/Informe Espa \%C3\%B1a completo red.pdf>. Acceso el: 31 ago. 2013.

GARRIDO, V. Antes que sea tarde. Cómo prevenir la tiranía de los hijos. Barcelona: Nabla, 2007.

GERARD, R. La agresividad, 2002. Disponible en: < http://www.aloja.cl/pdf/agresividad.pdf $>$. Acceso el: 27 ago. 2013.

GIMÉNEZ, A. M. Las dinámicas de Cyberbullying como ejemplo de exclusión educativa. En: COTRINA, M. J.; GARCÍA, M. (Coord.). Prácticas en Educación Inclusiva: diálogos entre Escuela, Ciudadanía y Universidad, 2012. p. 418-427.

GIMÉNEZ, A. M.; MAQUILÓN, J. J. Incidencia del Ciberbullying en los centros educativos de la Región de Murcia. En: MAQUILÓN, J. J.; MIRETE, A. B.; ESCARBAJAL, A.; GIMÉNEZ, A. M. (Coord.). Cambios educativos y formativos para el desarrollo humano y sostenible. Murcia: EDITUM, p. 449-458. 2011. 
GIMÉNEZ, A. M., ARNAIZ, P.; MAQUILÓN, J. J. Cyberbullying, la nueva cara de la violencia escolar. Alemania: Editorial Académica Española, 2013.

GÓMEZ, A.; GALA, F. J.; LUPIANI, M.; BERNALTE, A.; MIRET, M. T.; LUPIANI, S.; BARRETO, M. El bullying y otras formas de violencia adolescente. Cuaderno de Medicina Forense. 13 (48-49), p. 165-177, 2007.

KOWALSKI, R., LIMBER, S. Y AGATSTON, P. Cyber Bullying. El acoso escolar en la era digital. Bilbao: Desclée De Brouwer, 2010.

KUMPULAINEN, K.; RÄSÄNEN, E.; HENTTONEN, I. Children involved in bullying: Psychological disturbance and the persistence of the involvement. Child Abuse \& Neglect. 23 (12), p. 1253-1262, 1999.

LENHART, A. Cyberbullying and online teens. Pew Internet American Life Project, 2007. Disponible en: <http://www.pewinternet.org/ /media/Files/Reports/2007/PIP\%20Cyberbullying \%20Memo.pdf.pdf>.Acceso el: 31 ago. 2013.

LI, Q. Cyberbullying in schools. A research of gender differences. School Psychology International. 27 (2), p. 157-170, 2006.

MAQUILÓN, J. J.; GIMÉNEZ, A. M.; HERNÁNDEZ, F.; GARCÍA, A. Ciberbullying: Estudio de su incidencia en los centros educativos. En: ROMÁN, .M. J.; CARBONERO, M.A.; VALDIVIESO, J.D. Educación, Aprendizaje y Desarrollo en una sociedad multicultural. Madrid: Asociación de Psicología y Educación, p. 2781-2796. 2011a.

MAQUILÓN, J. J., GIMÉNEZ, A. M., HERNÁNDEZ, F. y GARCÍA, A.. La victimización en las dinámicas de ciberbullying en centros educativos de la Región de Murcia. International Journal of Developmental and Educational Psychology. 2, p. 265-275, 2011b.

McLAUGHLIN, J. H. Crime and punishment: Teen sexting in context. Peen State Law Review, 115 (1), p. 135-181, 2010.

MENÉNDEZ, I. Bullying: Acoso escolar, 2006. Disponible en: <http://www.psicopedagogia.com/bullying>. Acceso el: 27 ago. 2013.

MONTAÑÉS, M.; BARTOLOMÉ, R.; PARRA, M.; MONTAÑÉS, J. El problema del maltrato y el acoso entre iguales en las aulas. Revista de la Facultad de Educación de Albacete. 24, p. 1-13, 2009.

MORA, J. A.. Cyberbullying: un nuevo reto para la convivencia en nuestras escuelas. Información Psicológica. 94, p. 60-70, 2008.

MORALES, F. El efecto de la impulsividad sobre la agresividad y sus consecuencias en el rendimiento de los adolescentes, 2011. Tesis para optar al grado de doctor, Universitat Rovira i Virgili, Tarragona, España, 2007.

OBSERVATORIO DE LA SEGURIDAD DE LA INFORMACIÓN. Estudio sobre seguridad y 
privacidad en el uso de los servicios móviles por los menores españoles. Madrid: INTECO y Orange, 2010.

OBSERVATORIO DE LA SEGURIDAD DE LA INFORMACIÓN. Estudio sobre hábitos seguros en el uso de smartphones por los niños y adolescentes españoles. Madrid: INTECO y Orange, 2011.

OLWEUS, D. Conductas de acoso y amenazas entre escolares. Madrid: Morata, 2004.

OÑATE, A.; PIÑUEL, I. Informe Cisneros X: Acoso y Violencia Escolar en España. Instituto de Innovación Educativa y Desarrollo Directivo, 2007.

ORGANIZACIÓN MUNDIAL DE LA SALUD (OMS). Guía de bolsillo de la Clasificación CIE10. Madrid: Médica Panamericana, 2008.

ORTEGA, R. Víctimas, agresores y espectadores. Alumnos implicados en situaciones de violencia. Cuadernos de Pedagogía. 391, 2005. Disponible en: <http://www.pensamientocritico.org/rosort1104.htm>. Acceso el: 27 ago. 2013.

ORTEGA, R. La convivencia: un modelo de prevención de la violencia. En A. Moreno y M. P. Soler (coords.), La convivencia en las aulas: problemas y soluciones (29-49). Madrid: Ministerio de Educación y Ciencia, 2006.

ORTEGA, R.; MONKS, C. M. Violencia escolar y bullying. Información Psicológica, 87, 29-37, 2006.

ORTEGA, R.; MORA, J.; JÄGER, T. Actuando contra el bullying y la violencia escolar. El rol de los medios de comunicación, las autoridades locales e Internet. Landau, Germany: Verlag Empirische Pädagogik, 2007.

PELEGRÍN, A.; GARCÉS, E. J. Variables contextuales y personales que inciden en el comportamiento violento del niño. European Journal of Education and Psychology. (1 y 2)1, p. 520, 2008.

PRENSKY, M. Digital Natives, Digital Immigrants Part 1. On the Horizon. 9 (5), p. 1-6, 2001.

SMITH, P. K.; MAHDAVI, J.; CARVALHO, M.; TIPPETT, N. An investigation into Cyberbullying, its forms, awareness and impact, and the relationship between age and gender in Cyberbullying, 2006. Disponible <http://webarchive.nationalarchives.gov.uk/20130401151715/https://www.education.gov.uk/publica tions/eOrderingDownload/RBX03-06.pdf> . Acceso el: 27 ago. 2013.

TRIANES, M. V.; GARCÍA, A. Educación socio-afectiva y prevención de conflictos interpersonales en los centros escolares. Revista Interuniversitaria de Formación del Profesorado. 44, p. 175-189, 2002.

TORREGROSA, M. S.; INGLÉS, C. J.; GARCÍA, J. M.; RUIZ, C.; LÓPEZ, K. S.; ZHOU, X. Diferencias en conducta agresiva entre adolescentes españoles, chinos y mexicanos. European Journal of Education and Psychology. 3 (2), p. 167-176, 2010. 
VARJAS, K.; TALLEY, J.; MEYERS, J.; PARRIS, L.; CUTTS, H. High school student's perception of motivations for cyberbullying: an exploratory study. Western Journal of Emergency Medicine. 11 (3), p. 269-274, 2010.

WILLARD, N. Educator's guide to ciberbullying and cyberthreats. 2005. Disponible en: $<$ http://education.ohio.gov/getattachment/Topics/Other-Resources/School-Safety/Safe-andSupportive-Learning/Anti-Harassment-Intimidation-and-Bullying-Resource/Educator-s-GuideCyber-Safety.pdf.aspx>. Acceso el: 25 ago. 2013.

WILLARD, N. Cyberbullying and Cyberthreats: Effectively managing Internet use risks in schools, 2006.

WIREDSAFETY; AFTAB, P.. Cyberbullying: Methods and Types, 2008. Disponible en: < https://www.wiredsafety.org/wiredlearning/CB-Methods/index.htm>. Acceso el: 31 ago. 2013. 